\title{
Gradhiva
}

GRADHI

Revue d'anthropologie et d'histoire des arts

$23 \mid 2016$

Collections mixtes

\section{Collectionner par-delà nature et culture}

Julien Bondaz, Nélia Dias et Dominique Jarrassé

\section{(2) OpenEdition}

Journals

Édition électronique

URL : http://journals.openedition.org/gradhiva/3128

DOI : $10.4000 /$ gradhiva.3128

ISSN : 1760-849X

\section{Éditeur}

Musée du quai Branly Jacques Chirac

Édition imprimée

Date de publication : 25 mai 2016

Pagination : $28-49$

ISBN : 978-2-35744-093-7

ISSN : 0764-8928

Référence électronique

Julien Bondaz, Nélia Dias et Dominique Jarrassé, « Collectionner par-delà nature et culture », Gradhiva [En ligne], 23 | 2016, mis en ligne le 25 mai 2016, consulté le 24 septembre 2020. URL : http:// journals.openedition.org/gradhiva/3128; DOI : https://doi.org/10.4000/gradhiva.3128 


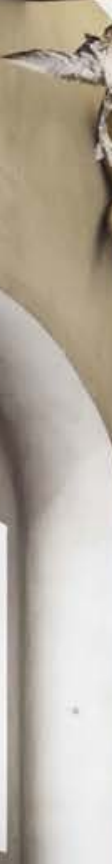

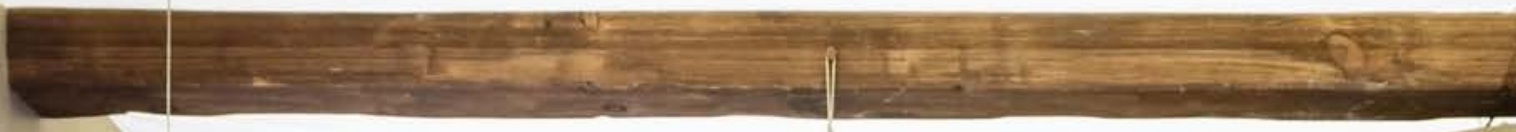

N

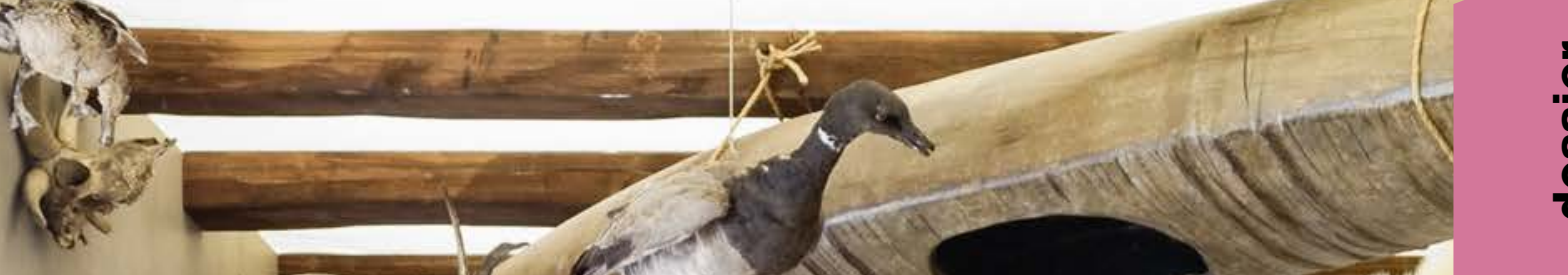

๖.
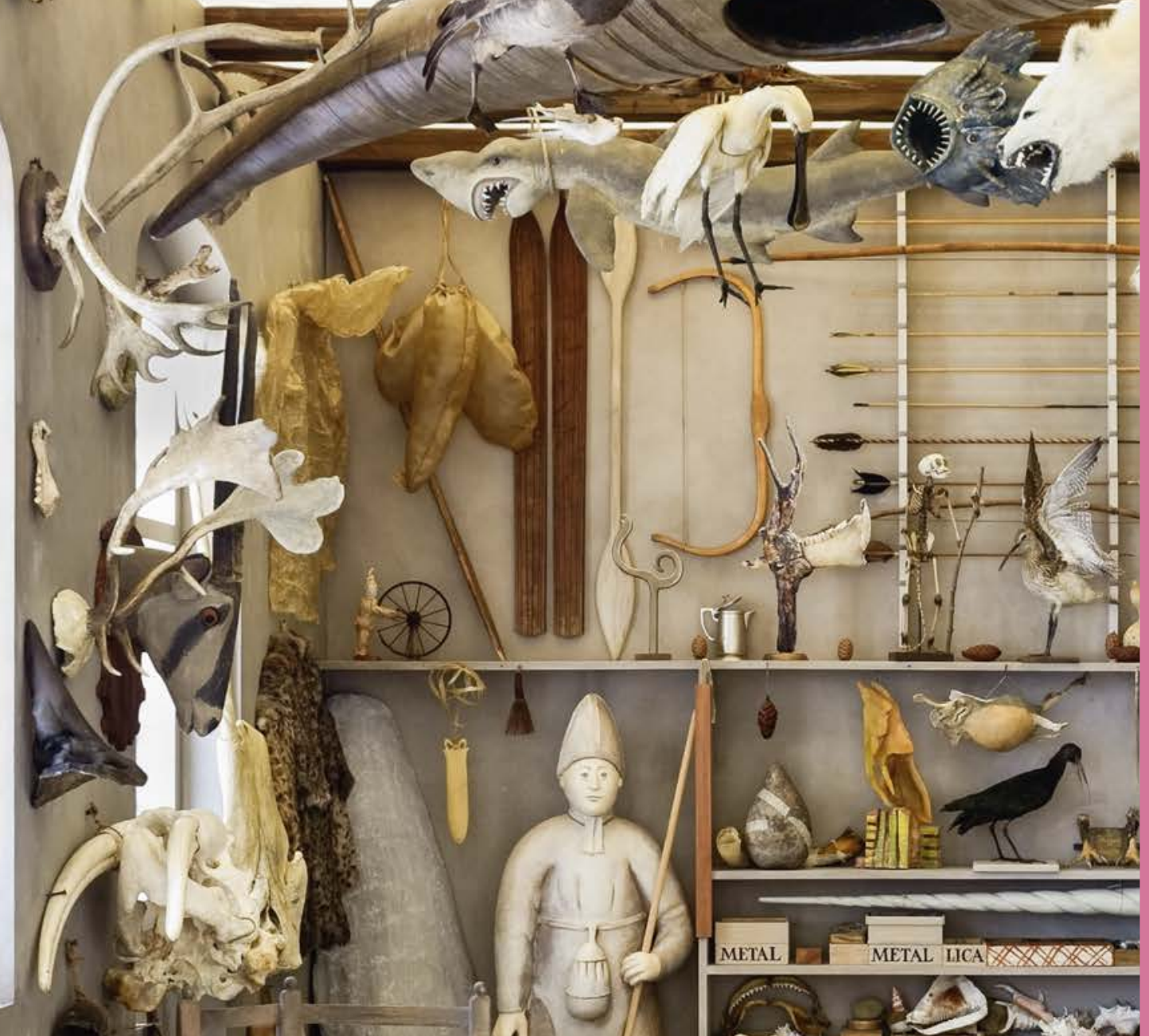

5) 31 (1)

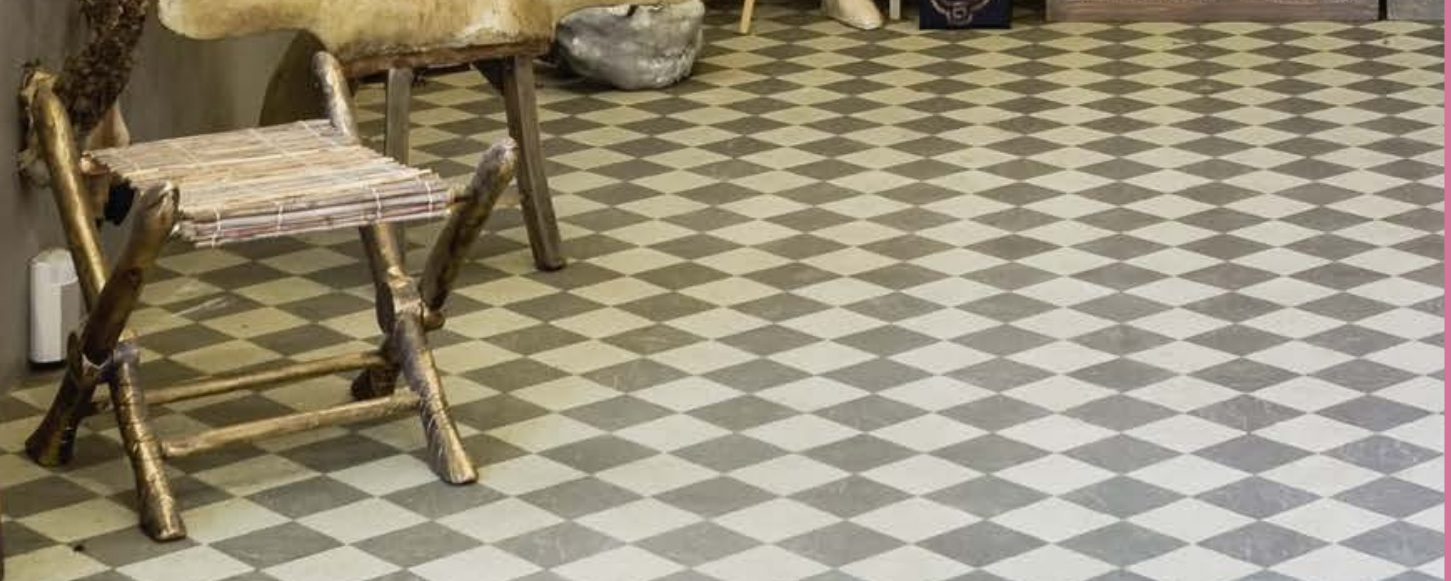




\section{Collectionner par-delà nature et culture}

par Julien Bondaz, Nélia Dias et Dominique Jarrassé

Les institutions muséales et les pratiques de collection constituent une porte d'entrée idéale pour étudier la vision du monde d'une société donnée. En 2001, en introduction d'un article intitulé «Par-delà la nature et la culture », Philippe Descola a proposé une description du musée de La Plata (capitale de la province de Buenos Aires) qui lui paraissait fournir «une excellente image du monde tel que nous l'avons longtemps conçu » (Descola 2001: 87). Dans ce musée s'opposent en effet un «rez-de-chaussée dévolu à la nature », où sont exposées les collections botaniques, zoologiques et minéralogiques, et un «étage de la culture», où les objets ethnographiques fonctionnent comme témoins matériels des peuples amérindiens ${ }^{1}$. Ce «microcosme à deux étages » fournit à l'auteur une métonymie inaugurale pour présenter l'ontologie naturaliste, puis les trois autres grandes ontologies qu'il considère au fondement de la diversité culturelle du monde. Descola concède cependant "que l'on s'emploie depuis pas mal de temps à frayer des passages entre les deux niveaux ", ceux de la nature et de la culture, ce qui n'est pas sans provoquer des résistances et des conflits. Le musée de La Plata paraît ainsi mettre en image le grand partage entre nature et culture, cadre du développement concomitant des musées modernes et des disciplines scientifiques.

La genèse du musée de La Plata témoigne cependant d'un cloisonnement plus relatif entre objets de la nature et artefacts humains. Dans l'Argentine de la seconde moitié du XIX siècle, Francisco Moreno, encore enfant, commence par rassembler une «imitation d'idole chinoise en terre cuite", des graines pétrifiées et un tas de cailloux brillants, un ensemble de coquillages ressemblant à «une patte de tigre pétrifiée», une étoile de mer et «deux balles de mitraille recueillies sur le champ de bataille de Waterloo» (Moreno 1890-1891; Barrio 1922). À l'âge de 14 ans, il crée son musée personnel, qu'il qualifie rétrospectivement de «musée bazar», en se référant au cabinet de curiosités de John Tradescant l'aîné, présenté à Lambeth, à Londres, au début du xvII ${ }^{\mathrm{e}}$ siècle ${ }^{2}$. Adulte, devenu explorateur, il ne cesse d'augmenter ses collections. En 1877, il fait don de son musée au gouvernement de la province de Buenos Aires, qui le déclare alors musée public sous le nom de Museo Antropológico y Arqueológico de Buenos Aires. En 1899, les collections sont transférées au musée de La Plata (la nouvelle capitale de la province ${ }^{3}$ ), qui est intégré sept ans plus tard à l'université nationale de la même ville. Les collections comptent alors plus de 200000 objets (environ 64000 pour la paléontologie, 50000 pour la zoologie, 40000 pour la géologie, 22000 pour la minéralogie et 20000 pour l'anthropologie et l'ethnographie), ce qui en fait le plus important musée d'Amérique du Sud. Dans le récit de création du musée de La Plata, la référence à Tradescant peut paraître anachronique: I'un des plus importants cabinets de curiosités du XVII siècle est proposé, plus de deux siècles plus tard, comme modèle muséal.
1. Cette organisation par étages (la nature au rez-de-chaussée, la culture au premier étage) se retrouve dans de nombreux musées à travers le monde, du Muséum d'histoire naturelle de Toulouse au Musée national du Kenya à Nairobi.

2. D'abord connu sous l'appellation «The Ark», il a été le premier musée britannique ouvert au public, sous le nom de Tradescant Museum, et est considéré comme un modèle (MacGregor [éd.] 1983).

3. On trouve à Buenos Aires (distante d'une soixantaine de kilomètres seulement de La Plata) un musée d'histoire naturelle plus ancien, créé en 1823 et connu aujourd'hui sous le nom de Musée argentin des sciences naturelles Bernardino Rivadavia. Malgré la proximité géographique des deux institutions, l'histoire de cet autre musée d'histoire naturelle est tout à fait différente de celle du musée de La Plata puisque ses collections archéologiques, qui voisinaient avec celles de zoologie, de botanique et de géologie, ont été transférées au musée d'ethnographie au milieu du $x x^{e}$ siècle. Sur l'histoire du musée de La Plata, voir González Pérez 2012 et Farro 2009. 


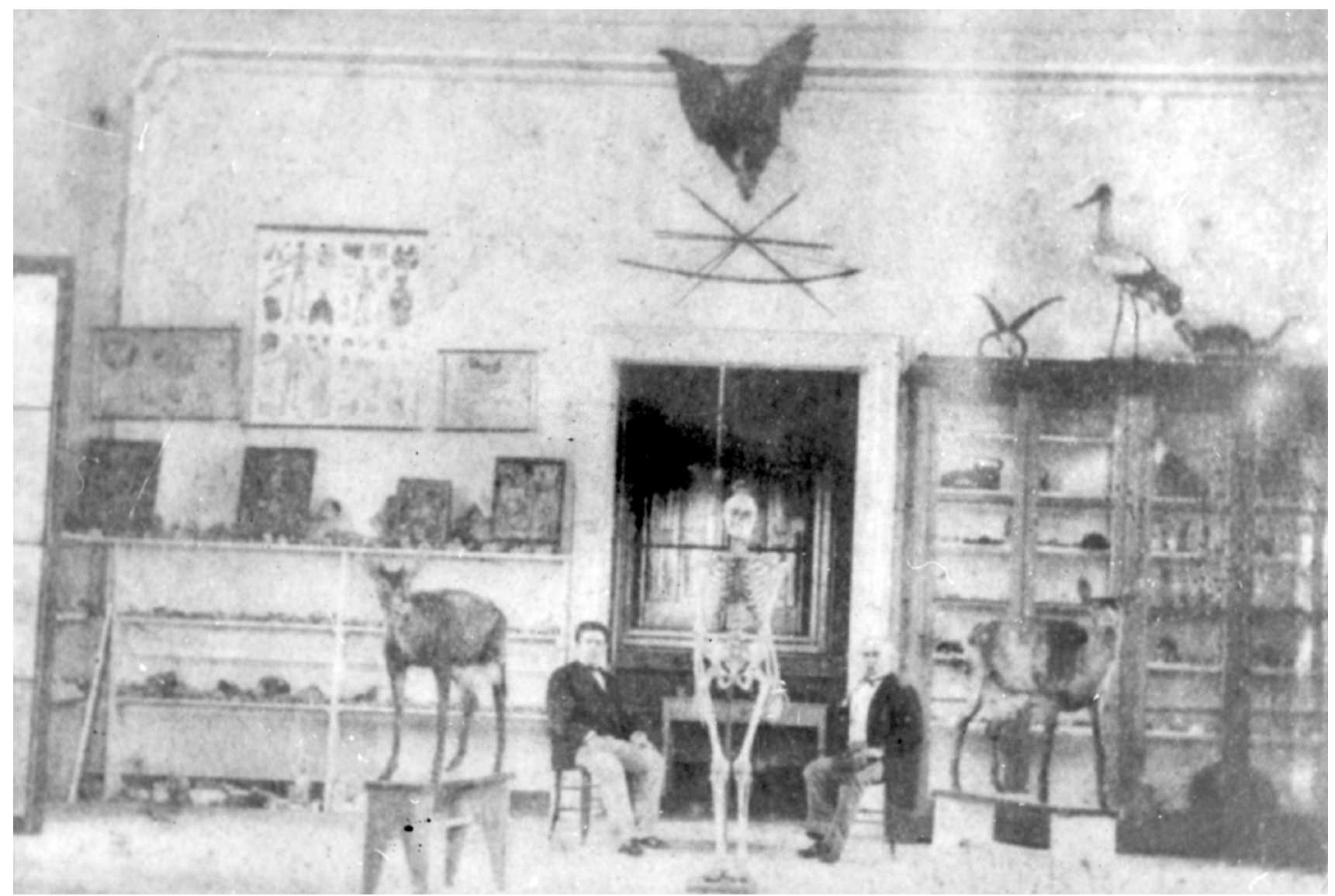

fig. 1

Francisco Moreno et son

père dans le musée de

la maison de campagne

familiale, vers 1872

(c) Archivo histórico

del Museo de La Plata. 
Ce qui se joue là est doublement intéressant. Le cas du musée de La Plata nous invite en premier lieu à nuancer l'idée selon laquelle la spécialisation et la disciplinarisation des musées au cours du XIX siècle auraient marqué la fin des cabinets de curiosités - et son corollaire: l'idée selon laquelle nous assisterions, depuis une vingtaine d'années, à une réactualisation de cette forme muséale (en art contemporain, dans la scénographie de plusieurs musées, au principe de nombreuses expositions temporaires, dans la littérature, pour ne citer que ces domaines ${ }^{4}$ ). Autrement dit, plutôt que de s'inscrire dans une perspective postmoderne ou d'imaginer ce que plusieurs auteurs anglo-saxons nomment le postmuseum (Hooper-Greenhill 2000), il paraît plus pertinent d'insister sur le fait que de nombreux musées et des activités de collection variées sont toujours restés réfractaires, pour diverses raisons, à la spécialisation et aux principes scientifiques de classification. Le modèle du cabinet de curiosités, qui fait l'objet depuis quatre décennies de recherches nombreuses et souvent renouvelées (voir en particulier Impey et MacGregor [éd.] 1985; Pomian 1993; Findlen 1994; Bredekamp 1996; Seipel [éd.] 2000; Falguières 2003; Le Fur 2006), n'a en réalité jamais cessé d'imprégner de nombreuses organisations muséales et doit être envisagé en termes de continuum du point de vue de la «généalogie des régimes muséologiques 5 » (Panese 2003).

Envisager la prégnance d'un tel modèle tout au long de l'histoire des musées ne permet cependant pas de comprendre de manière plus large comment perdurent, dans de nombreuses collections publiques ou privées, des rassemblements hétéroclites d'objets naturels et d'artefacts humains. Proposer de nommer "collections mixtes» de tels rassemblements permet d'insister sur le fait qu'ils ne respectent pas - voire remettent en cause - l'opposition entre nature et culture. Une telle qualification ne vaut sans doute que si l'on adopte les perspectives disciplinaires héritées du $\mathrm{XIX}^{\mathrm{e}}$ siècle, dont les critères de classification et de différenciation reposent sur les objets ou les champs d'étude. S'intéresser à l'existence de collections mixtes revient en effet à reconnaître qu'il en existe d'autres qui non seulement ne le sont pas, mais, qui plus est, reposent sur le principe d'une opposition entre spécimens naturels et artefacts humains ${ }^{6}$. La notion est donc relative: elle dépend des classifications en vigueur dans le contexte historique et culturel considéré et se révèle ainsi particulièrement mouvante. Dans cette mesure, toute classification muséale est une ethnoclassification que l'on peut analyser comme telle: collectionner revient à faire passer des objets d'un système de classification à un autre, d'une vision du monde à une autre. Il convient en effet d'être prudent ici en insistant sur un double biais, rétrospectif et ethnocentriste, qui semble sous-tendre l'idée de collection mixte entendue comme désignant les pratiques de collection ne distinguant pas (ou distinguant peu) les objets de la nature et les artefacts humains. La proposition se veut heuristique: il s'agit de contextualiser les effets d'hétéroclisme, les stratégies d'amalgames et les techniques de rassemblement qui interrogent en pratique, au sein des collections, le grand partage entre nature et culture. Il devient possible à cette condition d'écrire, au sujet de ces collections, une histoire qui se situe moins à la croisée des disciplines (Daugeron et Le Goff 2014) que dans une forme d'indiscipline, une «histoire mêlée - pour reprendre l'expression proposée par Isac Chiva à propos de la conjonction des patrimoines naturel et culturel (Chiva 2008).
4. Voir, par exemple, Davenne 2004.

\section{Sans parler plus} longuement ici des cas de permanence historique du cabinet de curiosités, comme au château d'Oiron (Martin, Guillaume et Didier 2000), ou des recréations déjà anciennes avec l'ouverture du musée Joseph-Denais à Beauforten-Vallée au début du $x x^{e}$ siècle.

6. La notion de «collection mixte " paraît préférable à celle de "collection composite " ou «hybride": elle rend mieux compte du dépassement de l'opposition entre deux grands ensembles d'objets qui nous intéresse ici. En outre, la notion de «collection hybride" est désormais d'usage spécifique: elle désigne (notamment dans les ouvrages anglophones dédiés à la gestion des musées, des archives et des bibliothèques) les collections qui rassemblent à la fois des objets physiques et des objets virtuels, mettant ainsi l'accent sur l'intégration des nouvelles technologies du numérique dans les institutions patrimoniales. 


\section{ci-contre}

fig. 2

Installation de l'artiste Rosamond Purcell, 2013 reprenant le cabinet de curiosité d'Ole Worm au Museum Wormianum en 1655. Photo Jens Astrup Natural History Museum of Denmark.
On suit ici les travaux des historiens des sciences qui visent à repenser la classification traditionnelle des disciplines (héritée du xIx ${ }^{e}$ siècle) en proposant une classification des savoirs nouvelle ayant pour critère les pratiques et les méthodes. II s'ensuit un rapprochement entre savoirs apparemment distincts en termes d'objet ou de champ d'étude, mais proches sur les plans méthodologique et épistémologique.

Le cas du musée de La Plata fournit en outre une première piste d'interprétation. Son histoire est en effet celle, classique, du passage de la collection privée au musée: on observerait ainsi une première phase, intime, de collecte mixte, suivie d'une seconde, publique, de mise en ordre muséale, et souvent de distribution disciplinaire des objets entre institutions muséales. Une telle conception des différences entre collectionneurs privés et conservateurs de musée, entre le caractère indiscipliné des collectes sur le terrain et la rigueur scientifique des cloisonnements muséaux, repose trop souvent sur une hiérarchie implicite entre les conservateurs ou les muséologues d'une part, et les collecteurs de terrain ou les collectionneurs privés d'autre part - voire sur une infantilisation ou une pathologisation des derniers au bénéfice des premiers. Parfois renversée, cette opposition peut être présentée comme une source d'inspiration: "À côté de l'ordre des musées, il y a le désordre des collectionneurs, insupportable pour l'historien dogmatique, mais révélateur dans la mesure où il génère des relations inattendues et fructueuses intellectuellement. » (Martin 2013: 16-17) Dans l'un et l'autre cas, penser les pratiques de collection privée et publique en termes d'opposition plutôt que d'articulation (Poulot 2011) empêche de comprendre, outre l'origine singulière de telle ou telle institution muséale, les continuités fortes qui existent entre ces deux types d'activité. Dans la perspective qui nous intéresse, la mixité des collections est très souvent commune aux collectionneurs privés et aux institutions muséales. Que ce soit dans les pratiques de collecte, dans l'agencement d'une collection personnelle chez soi ou dans les salles d'un musée public, les affinités entre spécimens naturels et artefacts humains produisent généralement les mêmes troubles cognitifs ou les mêmes effets esthétiques, c'est-à-dire les mêmes jeux de regard. Dans la mesure où les collections mixtes mêlent des produits humains et des œuvres de la nature, amalgament l'organique et l'inorganique, voire convoquent ensemble de l'animé et de l'inanimé, du vivant et des objets, elles donnent à voir des brouillages catégoriels, typologiques ou disciplinaires qui sont aussi ontologiques, et qui méritent d'être interrogés.

\section{Collectes indisciplinées}

Entre l'unification des collections de naturalia et d'artificialia au xvi ${ }^{e}$ siècle (Schnapper 1994: 10) et leur «partition » à partir de la fin du XVIII siècle (Daugeron 2009: 49), l'essor des chambres de merveilles (Wunderkammer) et des cabinets de curiosités est très généralement présenté comme un temps fort ou un âge d'or des collections mixtes. De nombreux travaux ont mis en évidence le caractère hybride de ces collections et l'importance du rôle qu'elles ont joué dans la production des savoirs à la fin de la Renaissance. La perspective encyclopédiste des collectionneurs, le «schème encyclopédique» de leurs pratiques (Findlen 1994) et leur visée microcosmique (Pomian 1988: 42; Lugli 1998) expliquent le caractère généralement composite de collections qui regroupaient naturalia et artificialia. Cependant, au cours du XVIII ${ }^{\mathrm{e}}$ siècle, 


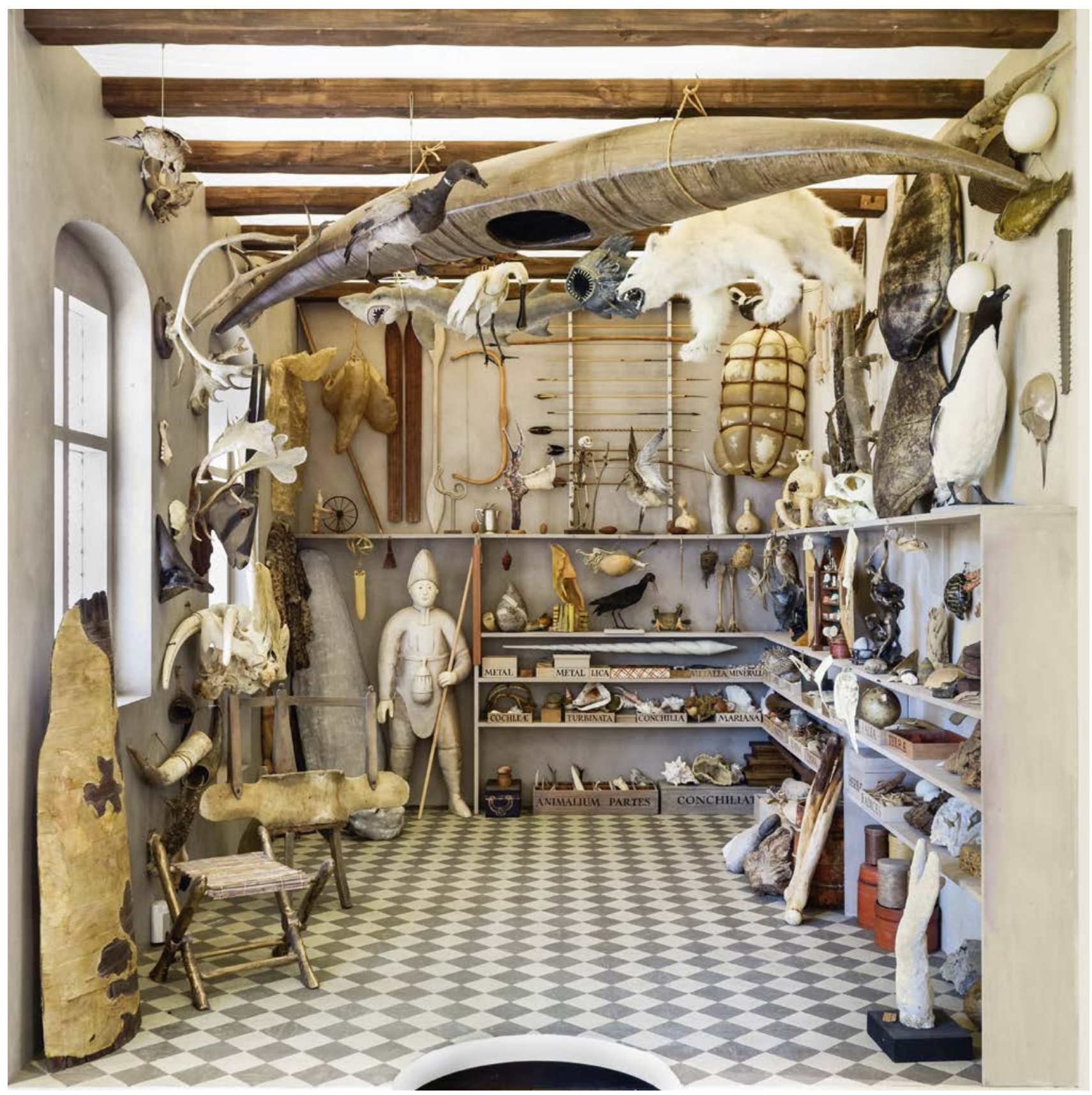




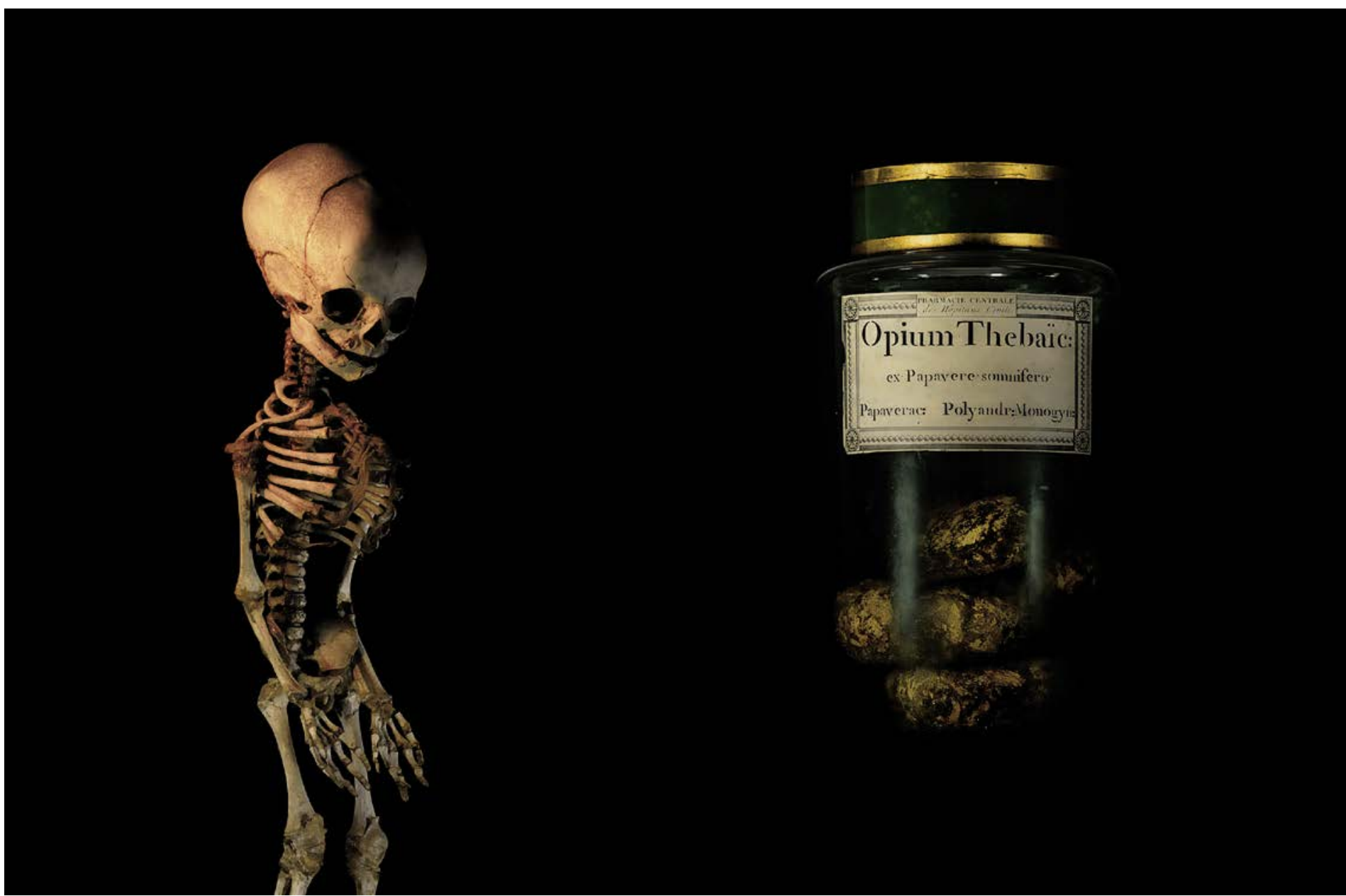

7. Si les anciennes civilisations de l'Amérique et de l'Asie étaient dès le début du XIX ${ }^{\ominus}$ siècle dans les musées d'art (au Louvre, notamment), celles de l'Afrique et de l'Océanie se trouvaient dans les musées d'histoire naturelle. Du point de vue de l'histoire des musées, la généalogie qui remonte jusqu'aux cabinets de curiosités fournit une part d'explication à cette mixité ancienne. C'est pour partie toujours le cas, avec des options variées en fonction des musées, qui vont du jeu sur le dispositif du cabinet de curiosités (comme au Muséum d'histoire naturelle de Toulouse) à l'exposition d'objets ethnographiques sous forme de transition entre aires géographiques (Muséum d'histoire naturelle de Rouen) Sur la place des collections ethnographiques dans les musées d'histoire

naturelle, voir Daugeron 2009.

8. Nous reprenons ici le titre du numéro 4 des Cahiers de l'École du Louvre, dirigé par Dominique Jarrassé. les collections encyclopédiques commencent à passer de mode. On assiste alors à une tendance générale de «mise en ordre des collections» (Pomian 2001), qui repose sur une normalisation et une standardisation des classifications scientifiques et muséales. Cette reconfiguration des frontières entre les objets de collection se traduit en particulier par la séparation des objets de la nature et des artefacts (à l'exception notable d'une partie de ceux des peuples «sauvages $\left.{ }^{7} »\right)$. Krzysztof Pomian, notamment, a bien montré comment l'histoire naturelle est alors passée «de la curiosité à la discipline», des cabinets au laboratoire et au terrain (Pomian 2004). Dans une perspective postmoderne qui, inspirée par les travaux de Michel Foucault, propose d'investir les différents sens du terme discipline, les musées postrévolutionnaires se retrouvent d'ailleurs analysés aujourd'hui comme des technologies disciplinaires (Hooper-Greenhill 1992; Bennett 1995). Ce mouvement de disciplinarisation, général au $\mathrm{XIX}^{\mathrm{e}}$ siècle, concerne aussi bien les sciences naturelles que les sciences de l'homme. Disciplinarisation des sciences et spécialisation des collections semblent aller de pair.

Mais l'étude des «modalités de collecte 8 » témoigne en réalité moins d'une rupture que d'une prégnance de l'hétéroclisme des objets collectés. Elle constitue en ce sens l'une des entrées privilégiées pour comprendre ce qui peut paraître indiscipliné dans un certain nombre de musées. L'idéal d'inventaire exhaustif et de saisie intégrale qui a animé les voyageurs naturalistes jusqu'au XIXe siècle, dans un souci de collecte du monde (Bourguet 1997), se rejoue en effet dans des missions de collecte postérieures. Le cas d'Achille 


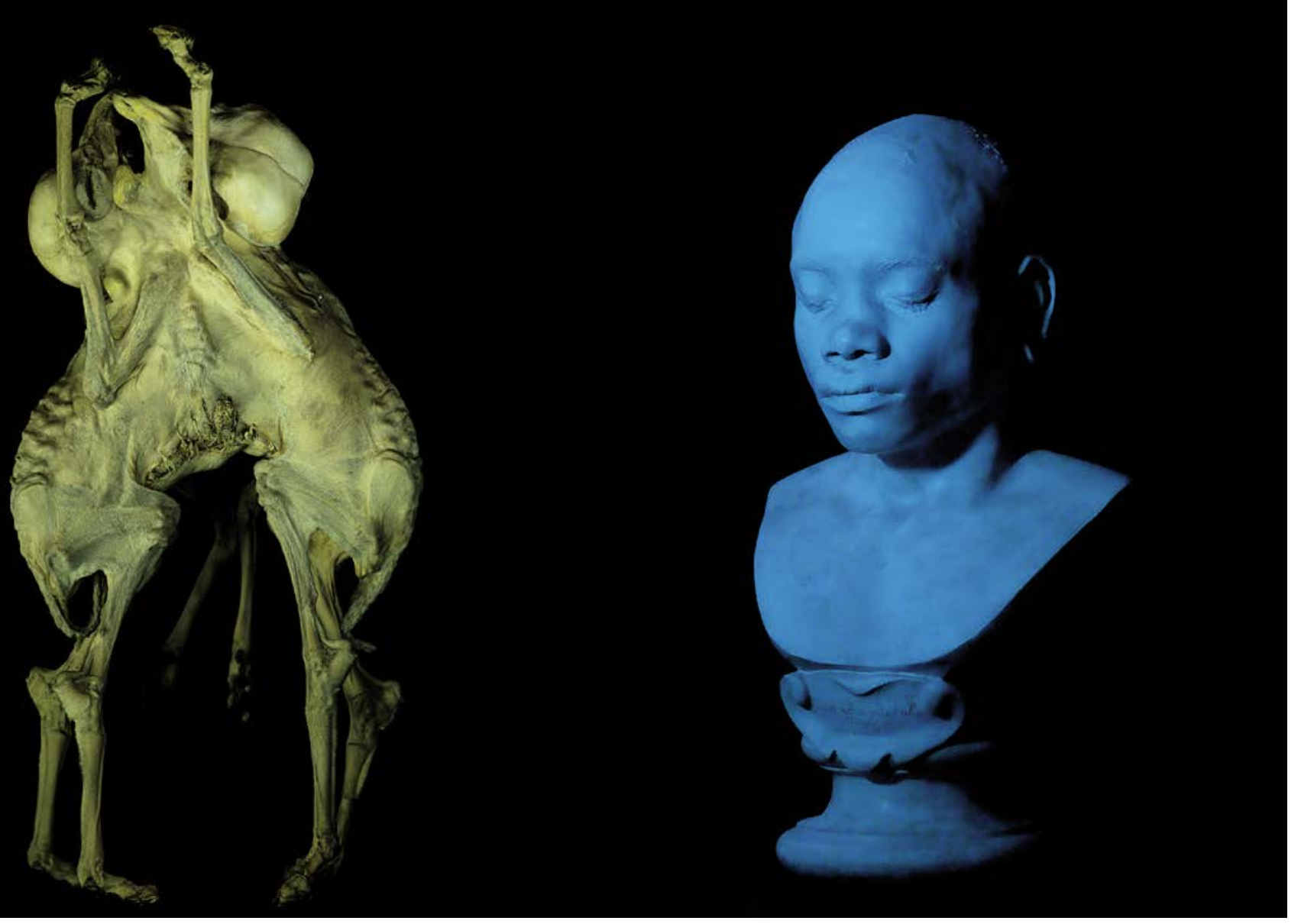

Raffray, entomologiste collecteur d'objets ethnographiques (Peltier, dans ce numéro), ou celui des missions organisées par le musée d'Ethnographie de Neuchâtel au début du xxe siècle (Reubi, dans ce numéro) ne sont pas exceptionnels. Entre les années 1930 et 1960, les collectes ethnographiques organisées en Afrique par le musée d'Ethnographie du Trocadéro puis le musée de l'Homme, à commencer par la fameuse mission Dakar-Djibouti, sont largement couplées à des collectes zoologiques, entomologiques et botaniques (Bondaz 2013 et 2015). À l'inverse, les exemples sont multiples de chasseurs passionnés par la faune exotique également collecteurs d'objets ethnographiques (Dias 1999) - à un point tel d'ailleurs que les trophées de chasse ont pu être envisagés comme un modèle de la collection de souvenirs (Pearce 1992: 69-72). L'un des cas les plus intéressants est peut-être celui du major Percy Horace Gordon Powell-Cotton, qui monte, après une carrière militaire, près d'une trentaine de missions de collecte en Afrique et en Asie, de la fin du xIx ${ }^{e}$ siècle presque jusqu'à sa mort en 1940. II diversifie peu à peu ses pratiques, ajoutant à la chasse aux mammifères un goût pour l'entomologie et un intérêt pour les objets ethnographiques, notamment pour les «fétiches» et les masques africains. Sa femme puis ses enfants poursuivent cette entreprise de collecte, mais remettent partiellement en question son caractère mixte: les objets ethnographiques sont recherchés par ses filles, les trophées de chasse rapportés par ses fils. Le Powell-Cotton Museum of African and Asian Zoology and Ethnography, fondé en 1896 dans une pièce de la résidence familiale, s'est rapidement agrandi et a ouvert au public trente ans après le décès de son initiateur (Nicklin 2001). fig. 3

Fiona Pardington.

We Dream of Gentle

Morpheus, 2011. Pigment

inks on Hahnemule Photo

Rag. With thanks to musée

du quai Branly, Paris. 
En outre, malgré la disciplinarisation des sciences au cours du $\mathrm{xI}^{\mathrm{e}}$ siècle et dans la première moitié $\mathrm{du} \mathrm{xx}^{\mathrm{e}}$ siècle, des similitudes méthodologiques peuvent être observées, pour certaines d'entre elles, à la fois au niveau des pratiques de collecte et dans la construction du terrain comme référent commun. Benoît de L'Estoile (2005) et Vincent Debaene (2006) ont notamment insisté sur le «paradigme de la collecte» dans la professionnalisation et la disciplinarisation de l'ethnologie. Plusieurs sciences naturelles et sciences de l'homme ont la collecte pour outil commun, la mise en exposition comme instrument partagé, permettant de mettre en évidence la comparaison, d'établir des typologies et de visualiser les connaissances. Robert $\mathrm{E}$. Kohler (2007) a ainsi proposé de regrouper les sciences naturelles, l'histoire de l'art, l'archéologie et l'ethnographie sous le nom de «sciences de la collecte", en soulignant le rôle fondamental joué par les pratiques de collecte et l'attention portée aux objets dans la production des savoirs au sein de ces disciplines. De même, Bruno Strasser a souligné la façon dont une histoire des sciences écrite à partir des pratiques, y compris les pratiques de collecte, pourrait amener à repenser les clivages disciplinaires, révélant ainsi «l'hétérogénéité des pratiques cognitives et matérielles au sein des disciplines et les similitudes entre les disciplines, alors que les histoires disciplinaires ont eu tendance à souligner les différences entre les disciplines et l'unité des pratiques cognitives et matérielles au sein des disciplines " (Strasser 2012: 309). La mise en exposition est d'ailleurs susceptible d'être travaillée par des tensions entre "sciences de la collecte", dans son principe comme dans ses dispositifs. Concernant les principes de classification et de mise en ordre muséographique, le débat qui a éclaté en Autriche à la fin du XVIII siècle en faveur d'un nouveau mode de présentation des œuvres d'art qui s'inspirerait des classifications naturalistes (Meijers 2001) a pour pendant, un siècle plus tard, en 1887, la polémique qui a opposé Franz Boas et Otis T. Mason: alors que le premier proposait d'appliquer le modèle des aires culturelles aux collections ethnographiques, le second défendait l'application du principe morphologique de la nomenclature linnéenne, en regroupant les objets selon leurs ressemblances formelles (Stocking 1974: 8-12). Plusieurs dispositifs de mise en exposition rendent par ailleurs compte d'une certaine polyvalence, qu'il s'agisse par exemple de la panoplie ou du diorama. Leur succès vaut autant pour les collections d'histoire naturelle que pour les artefacts humains.

\section{Musées mixtes}

La mixité des collections continue en effet de s'observer non seulement dans les pratiques de collecte, mais aussi dans les musées. Au xIx siècle, dans les principaux pays européens et en France en particulier, on constate une plus grande mixité des collections dans les musées provinciaux que dans ceux des capitales (Lacour, dans ce numéro). C'est dans ce contexte que l'appellation de "musée mixte" apparaît en France, dans une double logique de comparaison avec des propositions muséales étrangères et de concurrence entre nations, interprétée sous l'angle de la diversité des «mentalités» nationales. Dans la synthèse d'une conférence de Laurence Vail Coleman, directeur de l'American Association of Museums, publiée dans Le Bulletin de l'art en décembre 1927, on lit ainsi que "la distinction sévère, respectée généralement chez nous, entre les musées des beaux-arts, les musées d'histoire naturelle, les musées d'histoire échappe à la mentalité américaine: les musées neufs y sont la plupart du temps des musées 
mixtes». Un tel avis doit néanmoins être nuancé puisque, quelques années plus tard, en 1936, un recensement des musées français montre que les «musées mixtes» forment la catégorie la plus importante: sur 701 musées dénombrés, ils sont 270, contre 199 musées d'histoire, 157 musées d'art et 75 musées de science et de technique (L'Amour de l'art, $n^{\circ} 6$ ). L'expression désigne par la suite, dans la seconde moitié $d u x x^{e}$ siècle, les institutions muséales rattachées à deux ministères, ceux de la Culture et de l'Éducation nationale, du fait du caractère composite de leurs collections (la mixité désigne alors le statut administratif du musée et non directement les collections d'histoire naturelle et d'art ou d'histoire qu'il rassemble ${ }^{9}$ ).

Les principales institutions muséales où une telle mixité est observée sont évidemment celles dont la thématique se situe à l'articulation entre nature et culture et qui en rejettent, par certains aspects, le caractère dichotomique. Appliquée à ces musées, la notion d'objet-frontière, telle que les sociologues et les ethnologues l'ont développée (Trompette et Vinck 2009), rend bien compte à la fois de l'entre-deux dans lequel leurs collections se trouvent et des rencontres entre disciplines scientifiques auxquelles leur conservation et leur étude obligent. Cette notion, appliquée au sujet de l'importance de l'association entre professionnels et amateurs dans la production des savoirs naturalistes au sein d'un musée d'histoire naturelle (Star et Griesemer 1989), puis réinterrogée (entre autres exemples) à la lumière du développement des technologies du numérique comme outil de rencontre entre le social et le technique en contexte muséal (Meyer 2009), s'avère alors tout à fait heuristique. Dans cette perspective s'inscrivent par exemple le cas des musées de la chasse, qui interrogent le passage du domaine du sauvage à l'espace du domestique ${ }^{\mathbf{1 0}}$, en quoi réside précisément l'activité cynégétique, ou celui du «musée archéologico-technologique», dont Pomian a montré qu'il «rejette ainsi la coupure entre nature et culture» (Pomian 1988: 58). De même, les collections anthropologiques fournissent un cas d'étude désormais classique pour interroger la frontière entre nature et culture, l'ambivalence du statut des restes humains obligeant à des traitements muséaux tout à fait spécifiques (Berger 2008; Alberti, Bienkowski, Chapman et Drew 2009).

L'importance accordée à la mixité des collections s'explique également par la visée encyclopédiste qui continue de prévaloir dans certains musées, et en premier lieu dans ceux qui sont dotés d'un rôle éducatif. La multiplication des «musées scolaires » à partir du milieu du XIXe siècle, liée à la scolarisation d'un nombre d'enfants de plus en plus grand, s'inscrit dans une telle perspective. La liste des objets ou des images qu'ils doivent rassembler, pour servir de supports aux «leçons de choses», comporte une grande variété de spécimens naturels et d'artefacts humains (Vergnioux 2010). Les nombreuses descriptions du musée scolaire idéal sont autant de prescriptions pédagogiques pour les instituteurs, et mettent en avant la vertu des collections mixtes: «ll y aurait là de petites collections d'histoire naturelle, des tableaux d'histoire ou des objets d'art, des photographies de pays dont on lui parle dans les traités de géographie, des instruments de physique, de chimie, des tableaux astronomiques, des globes terrestres, des reproductions par la plastique de types ethniques. " (Trachsel 1898: 22) La conception de ces musées remet ainsi en question l'idée de collections scientifiques spécialisées:
9. L'expression récente de «musée hybride" fait également davantage référence à des modes d'organisation et d'administration des musées qu'aux collections qu'ils abritent (Mairesse 2012).

10. Voir les actes du colloque international Exposer la chasse (Paris, musée de la Chasse et de la Nature, 19-20 mars 2015) [en ligne], disponible sur: http://www. fondationfrancoissommer. org/fr/actualite. 
fig. 4

Vue de l'exposition Théâtre du Monde - la collection David Walsh, présentée à la Maison rouge du 19 octobre 2013 au 19 janvier 2014

(C) La Maison rouge photo Marc Domage.

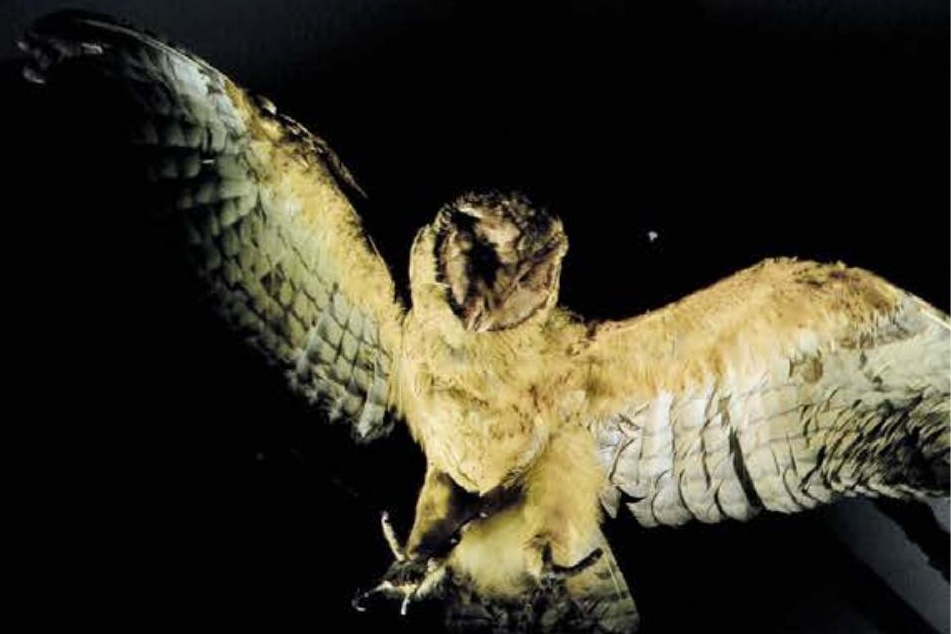

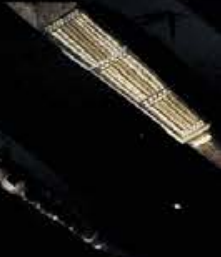

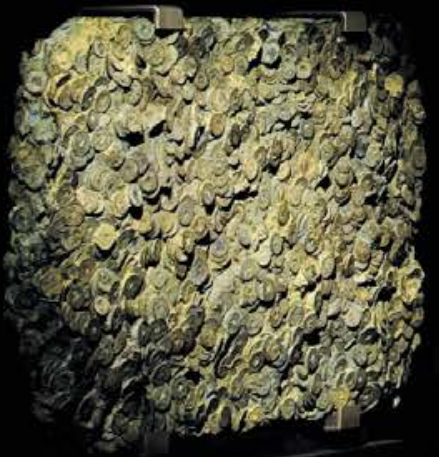




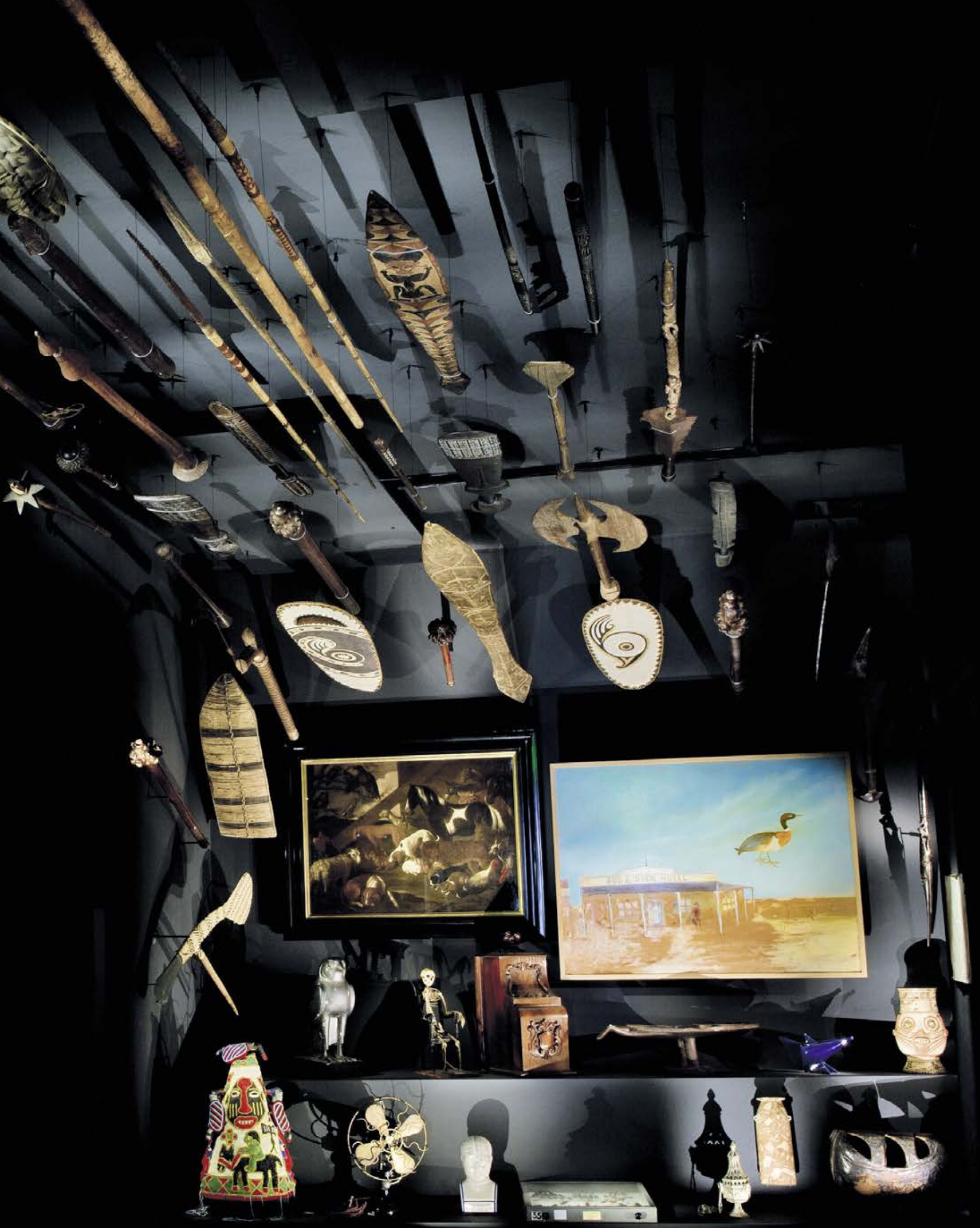




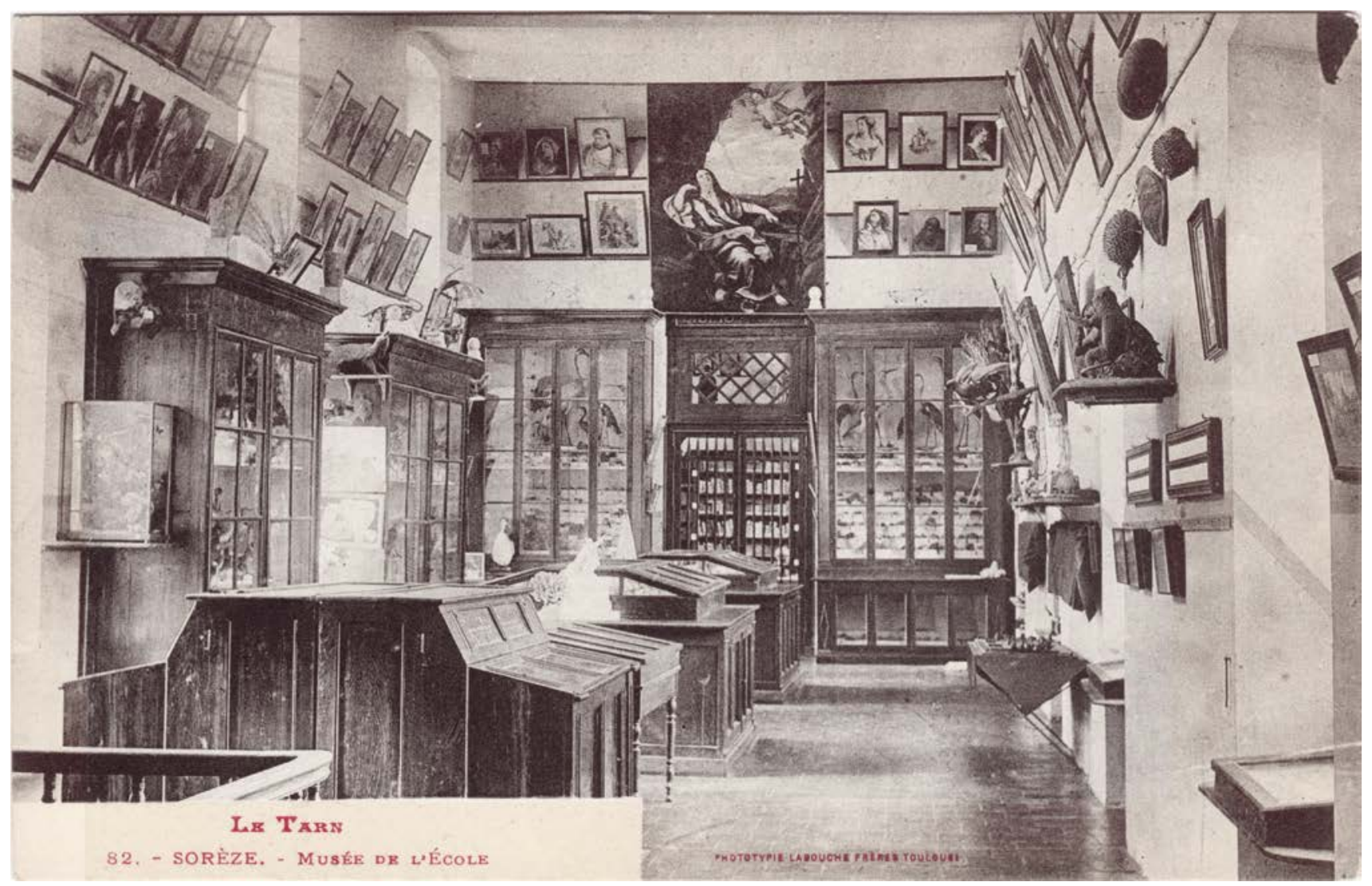

fig. 5

Le Tarn - Sorèze, Musée

de l'École, phototypie

Labouche, Toulouse,

carte postale ancienne

coll. part. 
le «musée bazar», version pour enfant du cabinet de curiosités, triomphe du «musée collection ${ }^{\mathbf{1 1}}$ ». Au début du xx siècle, ce modèle de musée scolaire se diffuse dans les colonies et rejoint celui des musées coloniaux.

Pendant la période coloniale, la création de musées dans les colonies (conjointement à celle de parcs zoologiques et botaniques) visait en effet la valorisation du territoire annexé. L'objectif était de présenter, sur place et prioritairement pour les colons, aussi bien les richesses naturelles (la faune, la flore, la géologie, et surtout les produits d'exportation) que la culture matérielle des différentes ethnies. Ces musées coloniaux avaient ainsi pour fonction de mettre en scène un «condensé de la colonie» (Dias 2000: 18). Quant aux musées coloniaux établis en métropole, ils ne pouvaient être strictement consacrés à une seule contrée, hormis le Congo pour la Belgique, les empires coloniaux s'étendant le plus souvent sur plusieurs continents. Un autre facteur explique leur nature mixte: leur origine comme musée commercial. La plupart d'entre eux ont été initialement constitués par des agences ministérielles et des chambres de commerce à partir de collections d'échantillons des produits (animaux, végétaux et minéraux) à exporter et commercialiser. Avant d'être des musées, ce furent des «instituts coloniaux» avec collections, bibliothèques, salles de presse et de conférence. Très vite s'est imposée l'idée d'une mise en scène qui contextualise les produits à l'aide de spécimens animaux naturalisés (avant qu'on ne se lance dans l'annexion de zoos) et d'artefacts locaux, voire de reconstitutions de milieux proches des aménagements des musées d'histoire naturelle; ainsi de la section coloniale de Tervuren lors de l'Exposition universelle de Bruxelles en 1897 (Jarrassé, dans ce numéro) ou de l'Exposition coloniale de 1906, présentée au Grand Palais et qui offrait grottes, animaux empaillés et mannequins... En France, l'institution centrale dans ce domaine était l'Exposition permanente des colonies qui offrait, dès 1855 , cartes, objets et spécimens, et les proposait aux expositions de province (Lozère 2009: 134-151); significativement, en 1899, fut aussi mis en place un jardin d'essai colonial à Vincennes. À Tervuren, tous les types de collections furent réunis par le roi Léopold II; le musée colonial ouvert en 1898 à l'issue de l'exposition était associé à un institut de recherche et à un zoo. La propagande prend le pas sur l'outil commercial, justifiant un investissement architectural et artistique majeur, et c'est un véritable palais qui est ouvert en 1910. Les débuts du musée colonial de Rome sont plus modestes et entièrement liés aux sciences naturelles (Gandolfo 2014). En 1904, il est créé comme Erbario e Museo Coloniale au sein de I'Istituto Botanico. En 1914, l'herbier est transféré à Florence et le musée au palais des Expositions, puis au Palazzo della Consulta (1923), siège du ministère des Colonies, pour s'installer enfin, en 1935 et en lien avec le projet impérial fasciste, dans un bâtiment en bordure du parc de la Villa Borghèse. Devenu essentiellement un musée historique, il retrouvait néanmoins une proximité significative avec le Giardino Zoologico fondé là en 1932. Bien d'autres fonctions sont donc assignées à ce musée qui accueille, par exemple, les canons italiens pris par les Éthiopiens lors de la défaite d'Adoua (1896) et repris en $1935 .$. . Par ailleurs, il reçoit des collections artistiques et organise régulièrement des expositions archéologiques ou d'artistes revenant des colonies. C'est là un trait des musées coloniaux des années 1930: ils instrumentalisent largement l'archéologie et l'art colonial.
11. Pour reprendre les expressions utilisées par un inspecteur d'académie en 1920. 
Dans plusieurs cas, comme on vient de le noter, la mise en scène du territoire conduit même à exposer des animaux vivants. Du cas le plus spectaculaire, celui de l'Exposition coloniale de Vincennes en 1931, il demeure aujourd'hui encore le palais de la Porte Dorée et le Parc zoologique de Vincennes (aujourd'hui Parc zoologique de Paris). Et mieux encore, au sein du palais de la Porte Dorée, qui a abrité successivement plusieurs musées, la présence originelle d'un aquarium et d'un terrarium n'a jamais été remise en cause. À l'époque où le palais abritait le musée des Arts d'Afrique et d'Océanie, deux tiers des visiteurs venaient pour les poissons et les sauriens, contre un tiers pour la statuaire africaine, et c'est donc à juste titre que les crocodiles ont été interprétés, en ouverture d'un livre consacré à la mémoire du musée, comme des «signes élevés entre science et art, entre collections vivantes et collections d'objets " (Eidelman, Monjaret et Roustan 2002: 9). Également héritier de la période coloniale et redéfini comme écomusée au moment des indépendances africaines, le Musée national Boubou Hama du Niger bénéficie grandement, lui aussi, de la curieuse mixité de ses collections: parce qu'il est à la fois un musée et un parc zoologique, parce que pavillons ethnographiques et archéologiques sont imbriqués dans les enclos et les bassins des bêtes, il est le musée qui rencontre aujourd'hui le plus grand succès populaire en Afrique de l'Ouest (Bondaz 2014).

Ce cas de musée colonial tardif transformé en écomusée, certes exceptionnel, est moins anodin qu'il n'y paraît: ces deux modèles muséaux ont en effet en commun un même but de valorisation territoriale. L'invention de l'écomusée (comme notion et comme forme d'exposition) témoigne de l'objectif d'exposer à la fois l'homme et son environnement, son territoire, c'est-à-dire «son foyer, son village, son petit pays, sa région, sa nation et, pourquoi pas, la terre entière " (Desvallées 2000: 12). II s'agit, pour reprendre le beau titre de Jean Davallon, de "claquemurer, pour ainsi dire, tout l'univers" (Davallon [dir.] 1986). Dans une telle logique, la mise en exposition du territoire obéit nécessairement à un principe de dépassement des distinctions entre nature et culture. Pour ce qui concerne l'importance accordée au territoire et l'idéal d'inventaire qu'ils défendent, les écomusées ont quelque chose à voir avec les musées coloniaux: alors que les seconds mettent en scène les territoires lointains des colonies, les premiers valorisent les proches espaces de la ruralité. Ils entretiennent les mêmes rapports que ceux qui se sont tissés, historiquement, entre les expositions universelles et coloniales, s'inscrivent dans une commune continuité. L'Exposition coloniale de 1931 et l'Exposition internationale de 1937 sont d'ailleurs l'une des inspirations, peu mise en avant, de Georges Henri Rivière. Mais c'est surtout dans le contexte de la création des parcs naturels, et en lien avec eux, que les écomusées (sous la première appellation de musées de plein air) sont inventés par le directeur du musée des Arts et Traditions populaires à la fin des années 1960 (Gorgus 2003: 260). Il s'agit pour Rivière de se détacher du «musée ethnologique de plein air » pour créer un «musée écologique de plein air » (Rivière 1971: 91).

Ce nouveau principe écologique, également au centre des missions de collecte initiées par Rivière et organisées en fonction d'«unités écologiques " (Desvallées 1989), témoigne d'un souci d'inventaire du territoire qui dépasse la distinction entre nature et culture. Le succès et la multiplication 


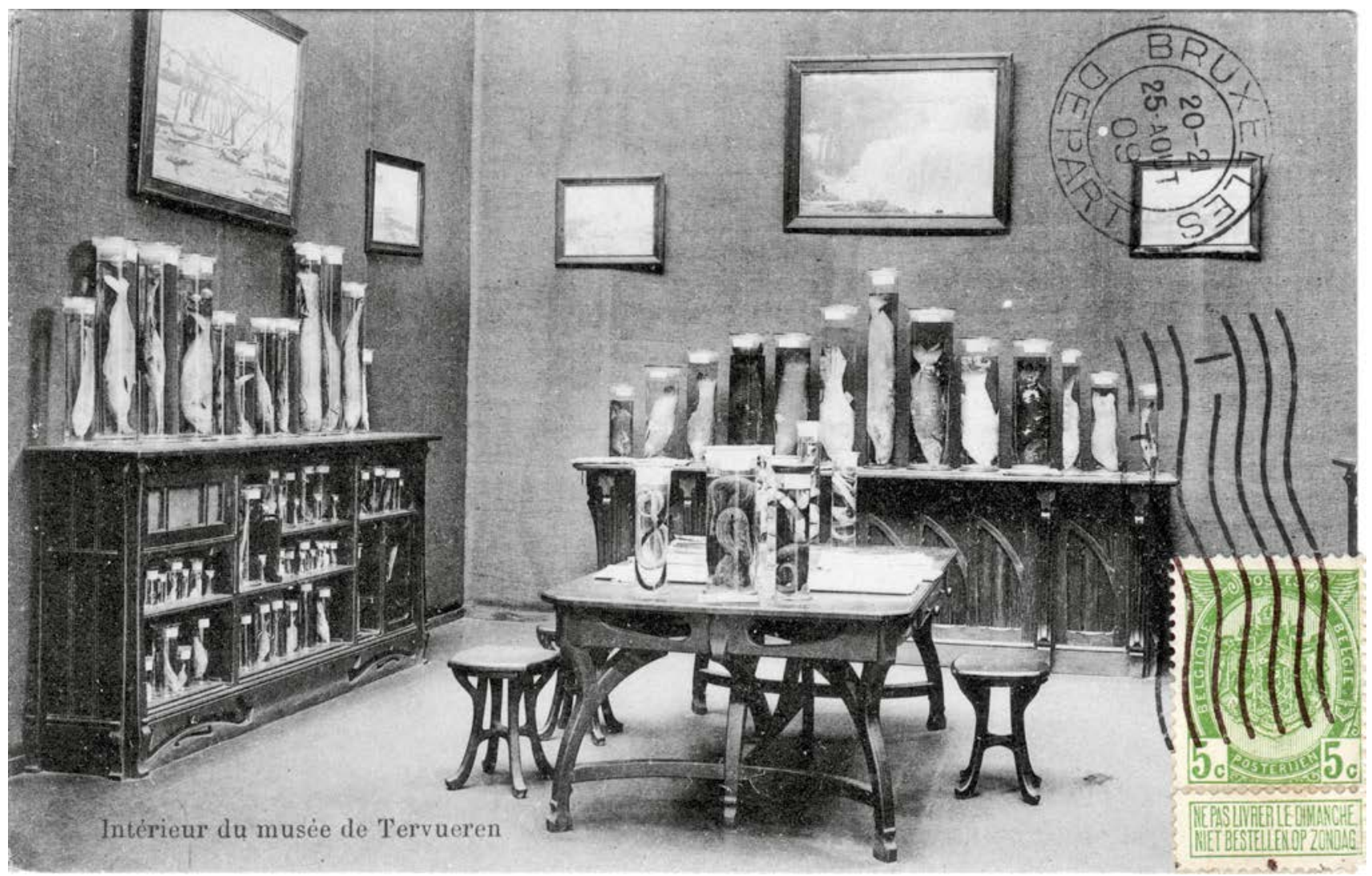

fig. 6

Intérieur du Musée de

Tervuren, spécimens

animaux, carte postale

ancienne, vers 1909

s. éd., coll. part. 
12. Entre autres références possibles on trouve dans l'œuvre de Gustave Flaubert la description de deux formes de collectionnisme, celui, naïf et nostalgique, de Félicité, la servante d'Un cœur simple, et celui, bourgeois et bonhomme, de Bouvard et Pécuchet, version ironique d'un encyclopédisme inachevé et d'une curiosité décadente (Bourgeois 2010). Flaubert note à propos de la chambre de Félicité qu'elle " avait l'air tout à la fois de chapelle et de bazar tant [l'endroit] contenait d'objets religieux et de choses hétéroclites": des chapelets, des médailles, des statuettes de la Vierge, un bénitier en noix de coco, une boîte en coquillages, des cahiers d'écriture, une géographie en estampes, des fleurs artificielles, un portrait du comte d'Artois, le tout rejoint par Loulou, le fameux perroquet.

13. Sur le développement du kitsch au xIx ${ }^{e}$ siècle, voir Olalquiaga 2002; sur les collections bourgeoises à la même époque, voir Charpy 2010. des écomusées dans les années 1970 ont ainsi contribué de manière durable à la remise en cause du caractère disciplinaire des collections et alimentent aujourd'hui encore les réflexions sur la mise en exposition des sciences. Dans cette perspective, les écomusées n'ont pas seulement pour fonction de donner à voir des valeurs communautaires ou des cultures locales: ils peuvent aussi avoir un impact sur les relations que les humains entretiennent avec leur environnement naturel (Magnusson 2004) ou remettre en question les modes de gouvernance de la nature (Borrelli et Davis 2012). Le modèle écologique dépasse le cadre des écomusées et invite à brouiller autrement encore l'opposition entre nature et culture. Dans les années 1970, au National Museum and Art Gallery du Botswana, il fut par exemple «décidé de renoncer à l'agencement classique en salles distinctes consacrées à l'ornithologie, ou à l'histoire, ou à la géologie, ou à la culture traditionnelle» et de présenter «une mosaïque qui mettrait en image l'homme replacé dans son environnement et dans son histoire, et qui mettrait également en relief les changements apportés à la terre » (Campbell et N'Teta 1980: 64). Si les formulations ont évidemment évolué depuis, la mise en exposition des interactions entre les humains et leur environnement est toujours d'actualité, comme en témoignent aussi bien l'ouverture du musée des Confluences (dont l'une des expositions temporaires inaugurales, La Chambre des merveilles, a d'ailleurs été consacrée aux cabinets de curiosités) que la refondation du musée de l'Homme.

Du point de vue de l'histoire des musées, trois facteurs principaux expliquent donc la persistance du caractère mixte de nombreuses collections: la visée pluridisciplinaire qui transforme les collections muséales en objets-frontières, le schème encyclopédiste qui continue de soutenir les discours éducatifs et propagandistes, et enfin le principe monographique ou écologique qui propose de mettre en scène l'ensemble des richesses ou des aspects d'un territoire, dont les musées coloniaux et les écomusées sont sans doute, chacun à leur façon, les deux formes les plus abouties. On aurait tort cependant de ne pas souligner les effets esthétiques et les inspirations artistiques produits par ces rassemblements d'objets hétérogènes, qui ne s'expliquent pas seulement par des processus de légitimation scientifique.

\section{Collections hétéroclites}

Les collections privées du $\mathrm{XIX}^{\mathrm{e}}$ siècle, dont la littérature de l'époque a souligné l'aspect souvent hétéroclite ${ }^{\mathbf{1 2}}$, témoignaient d'une esthétique rétrospectivement qualifiée de kitsch et produite autant par certains des bibelots rassemblés que par l'effet procuré par leur voisinage, brouillant les distinctions entre objets de la nature et produits manufacturés (boîtes en coquillages ou fleurs artificielles, par exemple ${ }^{\mathbf{1 3}}$ ). Les artistes des avantgardes, à commencer par les surréalistes, amateurs comme on le sait de rencontres fortuites et souvent collectionneurs eux-mêmes, se sont également confrontés à la distinction entre produits de la nature et artefacts humains. La célèbre Exposition surréaliste d'objets d'André Breton, en 1936, qui regroupait objets naturels, trouvés ou interprétés, objets perturbés, objets d'Océanie et d'Amérique du Nord, objets mathématiques, objets surréalistes, est exemplaire, tout comme son fameux «mur» installé après la seconde guerre mondiale dans son appartement parisien, où des boîtes de papillons et de cigales, des pierres et un os de baleine 
voisinaient avec une amulette égyptienne, deux masques tatanua et iroquois, des œuvres de Joan Miró, Francis Picabia, Jean Degottex et René Duvillier (Dufour 2004). De telles installations se retrouvent dans nombre d'œuvres d'art contemporain, qui entrent d'autant plus difficilement en collection qu'elles intègrent des matières organiques, vivantes, donc périssables (Bret, dans ce numéro).

L'exposition et le mur de Breton continuent par ailleurs de servir de référence plus ou moins explicite à nombre de propositions muséales ou d'expositions. On peut notamment penser aux expositions d'Yves Le Fur - Résonances, qui a mis en valeur la collection du Dr Jean-Claude Andrault, collectionneur d'art africain et d'objets naturels trouvés, au musée Dapper en 1990, et La Nature à l'œuvre, en 2000 -, à N'importe quoi, présentée au musée d'Art contemporain de Lyon, en 2009, et au Théâtre du monde de Jean-Hubert Martin, à la Maison rouge fin 2013-début 2014. Plus récemment encore, l'exposition Formes simples, montée en 2015 au Centre PompidouMetz par Jean de Loisy, rapprochait «des sujets industriels, mécaniques, mathématiques, physiques, biologiques, phénoménologiques ou archéologiques avec des objets d'art et d'architecture, tout en confrontant ceux-ci à leurs ancêtres archaïques et à des objets naturels". Dans tous les cas, déconstruire la mise en ordre du monde revient à bouleverser un certain ordre esthétique, une hiérarchie des valeurs, et donc à interroger le statut de l'œuvre d'art et les dispositifs de sa mise en exposition. Comme l'a proposé Martin, il s'agit de passer d'un «musée docile» à un «musée des charmes» (Martin 2013), d'abandonner les normes classificatoires pour provoquer des rapprochements inopinés producteurs d'effets et qui valent avant tout pour celui qui regarde. Ce glissement de l'empire de la raison au règne des émotions, de la cohérence des objets à l'expérience du sujet, signale à coup sûr une personnalisation des collections, un saisissement: pour le collectionneur, l'artiste ou le commissaire d'exposition, l'enjeu n'est plus seulement de collecter le monde, mais de l'éprouver.

On comprend mieux pourquoi la mixité des collections s'est davantage maintenue dans les collections privées que dans les musées: elles n'ont pas à se conformer aux mêmes principes de mise en ordre et de lisibilité que les collections muséales. Contrairement à ces dernières, les collections privées ne doivent pas respecter "les impératifs d'un classement prétendant à une validité intersubjective " (Pomian 2001: 18), mais valent davantage comme œuvre personnelle. Dans ces pratiques de collection mixte se mêlent souvent des recherches d'effets esthétiques et des ressorts biographiques. Les cas de collectionneurs privés amassant indifféremment des objets de la nature et des artefacts humains sont multiples, depuis Charles-Louis-Fleury Panckoucke, figure emblématique du collectionneur des années 1830, qui rassembla aussi bien des œuvres d'art ou des antiquités que des coquillages, jusqu'au sculpteur et collectionneur catalan Frederic Marès, dont l'étonnant musée barcelonais regroupe des sculptures, des objets du quotidien et des compositions naturalistes, ou au collectionneur allemand Thomas Olbricht, qui inscrit sa démarche dans la filiation des cabinets de curiosités. Dans un autre registre, le cas de Jacques Kerchache, collectionneur d'art premier et d'insectes, de papillons notamment, à l'origine du musée du quai Branly, est lui aussi bien connu. 


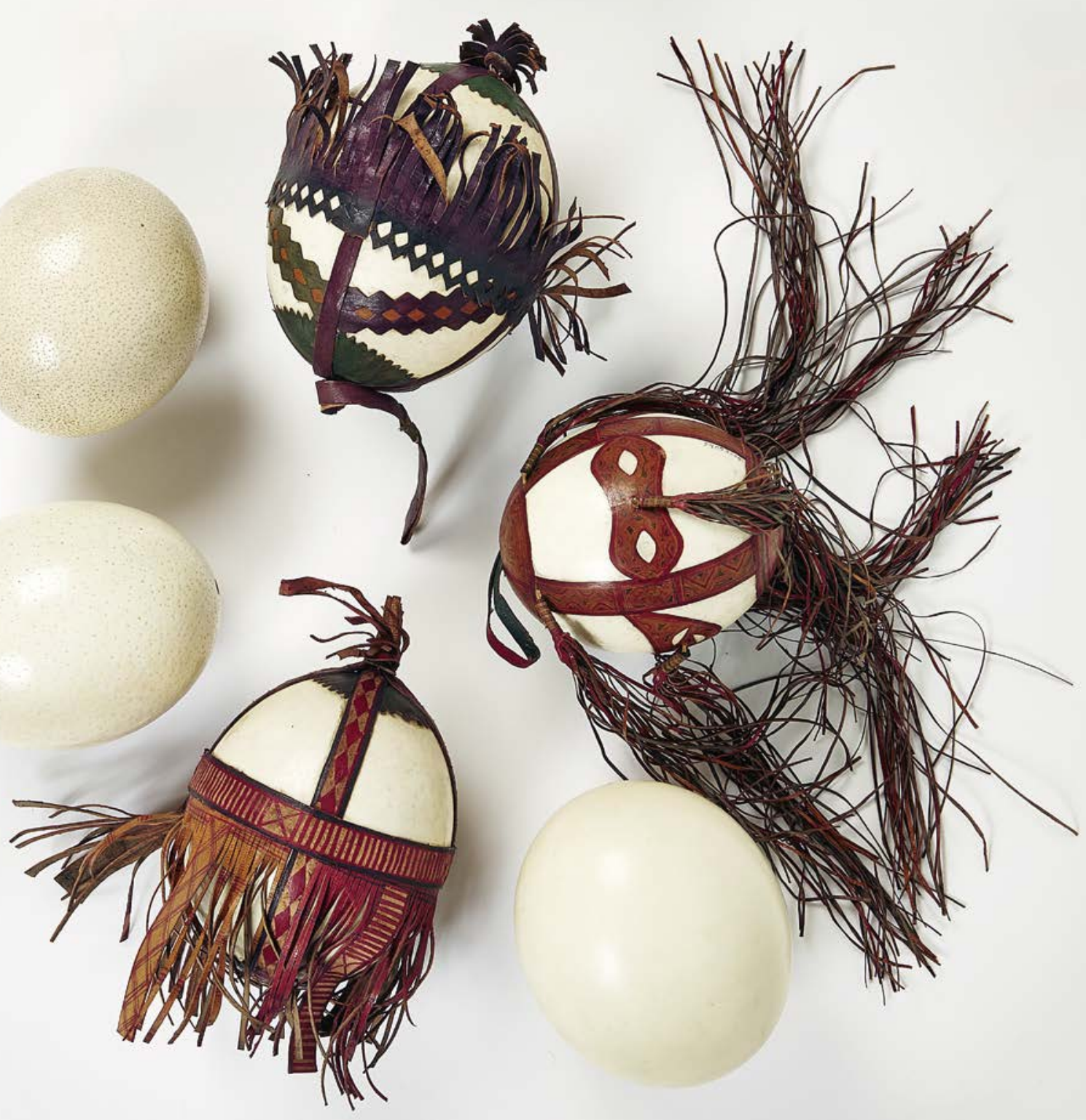


Cette conjonction de goûts, que l'on retrouve chez plusieurs collectionneurs d'art premier (Derlon et Jeudy-Ballini, dans ce numéro), peut évidemment interroger. On peut ainsi se demander dans quelle mesure les collections mixtes n'ont pas pour effet de naturaliser les artefacts et les humains qui les ont produits.

Le plaisir esthétique trouve cependant une autre justification dans le discours de Kerchache. Dans le catalogue consacré à sa collection entomologique, une citation de Claude Lévi-Strauss était reprise: «Un oiseau, un scarabée, un papillon invitent à la même contemplation fervente que nous réservons au Tintoret ou à Rembrandt. " (Lévi-Strauss et Eribon 2009: 241) En écho à cette rencontre entre les petites bêtes et les toiles des plus grands maîtres de la peinture occidentale, dans le lot de critiques que l'anthropologue avait formulées contre le musée d'Orsay lors de son ouverture se trouvaient ciblées des salles où «les tableaux semblent en cage dans quelque jardin zoologique " (Lévi-Strauss 1987: 181). Le grand partage entre nature et culture, que Lévi-Strauss a pourtant contribué à entériner dans les sciences sociales, se retrouve ici perturbé à la fois par son goût indiscipliné pour la beauté du monde et par son expérience critique des musées. Comme lui, nombre d'amateurs d'art, de visiteurs d'expositions, de collectionneurs ou de conservateurs ne cessent en réalité de frayer des passages dans la diversité des choses collectées entre les œuvres naturelles et culturelles, remixant les savoirs et les émotions, brouillant les balises ontologiques et les pistes muséales.

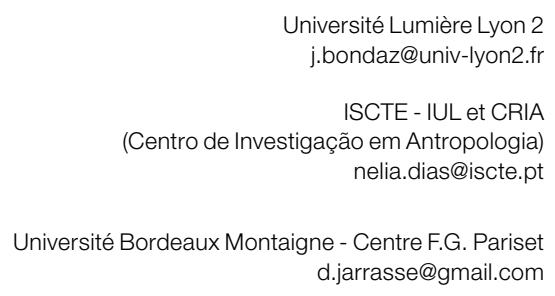




\section{Alberti, Samuel J.M.M., Bienkowski, Piotr, Chapman, Malcom J. et Drew, Rose \\ 2009 "Should we display the dead? ", Museum \& Society 7(3): 133-149.}

\section{Barrio, Maximino de}

1922 "Le musée de La Plata; les trois époques", Journal de la Société des américanistes 14-15: 342-349.

\section{Bennett, Tony}

1995 The Birth of the Museum. History, Theory, Politics. Londres et New York, Routledge.

\section{Berger, Laurent}

2008 "Des restes humains trop humains", La viedesidées.fr, [en ligne], disponible sur: http://www.laviedesidees.fr/Desrestes-humains-trop-humains.html.

\section{Bondaz, Julien}

2013 "L'ethnographie parasitée? Anthropologie et entomologie en Afrique de l'Ouest (1928-1960)", L'Homme 206: 121-150.

2014 L'Exposition postcoloniale. Musées et zoos en Afrique de l'Ouest. Paris, L'Harmattan.

2015 «Bêtes de terrain. Savoirs et affects dans l'invention de l'ethnozoologie", Anthropologie et Sociétés 39(1-2) : 37-59.

\section{Borrelli, Nunzia} et Davis, Peter

2012 "How culture shapes nature: Reflections on ecomuseum practices", Nature and Culture 7 : $31-47$.

\section{Bourgeois, Bertrand}

2010 "Le Muséum de Bouvard et Pécuchet. Parodie, kitsch et ruine du musée moderne", French Literature Series 37(1): 99-111.

\author{
Bourguet, Marie-Noëlle \\ 1997 «La collecte du monde: \\ voyage et histoire naturelle \\ (fin XVII" siècle-début XIXe siècle) ", \\ in Claude Blanckaert, Claudine \\ Cohen, Pietro Corsi et Jean-Louis \\ Fischer (coord.), Le Muséum \\ au premier siècle de son histoire. \\ Paris, Muséum national d'histoire \\ naturelle: 163-196.
}

\section{Bredekamp, Horst}

1996 La Nostalgie de l'antique. Statues, machines et cabinets de curiosités. Paris, Diderot.

\section{Campbell, Alexander Colin et N'Teta, Doreen}

1980 «Musée national et Galerie d'art, Botswana: historique et perspectives ", Museum International 32(1-2) : 62-67.

\section{Charpy, Manuel}

2010 Le Théâtre des objets. Espace privé, culture matérielle et identité bourgeoise. Paris 1830-1914. Université de Tours, thèse de doctorat.

\section{Chiva, Isac}

2008 "Sciences de l'homme et patrimoines naturels", Techniques \& Culture 50: 16-39.

\section{Daugeron, Bertrand}

2009 "Classement et rangement des "objets des sauvages" vers 1800. L'ordre méthodique comme écriture des objets ", Culture \& Musées 14(1): 39-63.

\section{Daugeron, Bertrand et Le Goff, Armelle}

2014 Penser, classer, administrer. Pour une histoire croisée des collections. Paris, CTHS-MNHN.

\section{Davallon, Jean (dir.)}

1986 Claquemurer, pour ainsi dire, tout l'univers. La mise en exposition. Paris, Centre de création industrielle-Centre Georges-Pompidou.

\section{Davenne, Christine}

2004 Modernité du cabinet de curiosités. Paris, L'Harmattan.

\section{Debaene, Vincent}

2006 «"Étudier des états de conscience" : la réinvention du terrain par l'ethnologie, 19251939 ", L'Homme 179: 7-62.

\section{Descola, Philippe}

2001 «Par-delà la nature et la culture», Le Débat 114: 86-101.

\section{Desvallées, André}

1989 "Collecte en Aubrac ", in Collectif, La Muséologie selon Georges Henri Rivière. Paris, Dunod: 185-187.

2000 «Introduction", Publics et musées 17-18 ("L'écomusée: rêve ou réalité "): 11-31.

\section{Dias, Nélia}

1999 "L'Afrique naturalisée ", Cahiers d'études africaines 155-156: 583-594.

2000 "Musées et colonialisme: entre passé et présent ", in Dominique Taffin (textes réunis par), Du musée colonial au musée des cultures du monde. Paris, Musée national des Arts d'Afrique et d'Océanie-Maisonneuve et Larose: 15-33.

\section{Dietz, Bettina et Nutz, Thomas}

2005 «Collections curieuses: The aesthetics of curiosity and elite lifestyle in Eighteenth-Century Paris ", Eighteenth-Century Life 29(3): 44-75.

\section{Dufour, Bernard}

1994 Des collectionneurs te/s André Breton. Saint-Clémentde-Rivière, Fata Morgana.

\section{Eidelman, Jacqueline, Monjaret, Anne et Roustan, Mélanie \\ 2002 MAAO. Mémoires. Paris, Marval.}

\section{Falguières, Patricia}

2003 Les Chambres des merveilles. Paris, Bayard.

\section{Farro, Máximo}

2009 La formación del Museo de La Plata. Coleccionistas, comerciantes, estudiosos y naturalistas viajeros a fines del siglo XIX. Rosario, Prohistoria Ediciones.

\section{Findlen, Paula}

1994 Possessing Nature. Museums, Collecting, and Scientific Culture in Early Modern Italy. Berkeley,

University of California Press.

\section{Gandolfo, Francesca}

2014 /l Museo Coloniale di Roma (1904-1971). Fra le zebre nel paese dell'olio di ricino. Rome,

Gangemi Editore.

\section{González Pérez, Carlos Federico}

2012 "Referencias históricas del Museo de La Plata, Facultad de Ciencias Naturales y Museo. Trayectorias necesarias para entender su presente ", Aletheia 3(5) [en ligne], disponible sur: http:// www.aletheia.fahce.unlp.edu.ar.

\section{Gorgus, Nina}

2003 Le Magicien des vitrines. Paris, Éditions de la Maison des sciences de l'homme.

\section{Hooper-Greenhill, Eilean}

1992 Museums and the Shaping of Knowledge. Londres et New York, Routledge.

\section{Museums and the} Interpretation of Visual Culture. Londres et New York, Routledge.

\section{Impey, Oliver et} MacGregor, Arthur (éd.) 1985 The Origins of Museums. The Cabinet of Curiosities in Sixteenth-and SeventeenthCentury Europe. Oxford, Clarendon Press.

\section{Kohler, Robert E.}

2007 «Finders, keepers: Collecting sciences and collecting practices", History of Science 45: 428-454. 


\section{Le Fur, Yves}

2006 D'un regard l'autre. Histoire des regards européens sur l'Afrique, l'Amérique et l'Océanie. Paris, musée du quai Branly-RMN.

\section{L'Estoile, Benoît de}

2005 « "Une petite armée de travailleurs auxiliaires" : la division du travail et ses enjeux dans l'ethnologie française de l'entredeux-guerres ", Cahiers du Centre de recherche historique 36 :

31-59 [en ligne], disponible sur: http://ccrh.revues.org/3037.

\section{Lévi-Strauss, Claude}

1987 "Le cadre et les œuvres", Le Débat 44: 180-183.

\section{Lévi-Strauss, Claude et Eribon, Didier}

2009 De près et de loin. Paris,

Odile Jacob.

\section{Lozère, Christelle}

2009 Mises en scène de l'objet dans les "salons coloniaux" de province (1850-1896). Vers l'émergence de modèles d'expositions coloniales. Université Bordeaux Montaigne, thèse de doctorat.

\section{Lugli, Adalgisa}

1998 Naturalia et Mirabilia: les cabinets de curiosités en Europe. Paris, Adam Biro.

\section{MacGregor, Arthur (éd.)}

1983 Tradescant's Rarities: Essays on the Foundation of the Ashmolean Museum (1683), with a Catalogue of the Surviving Early Collections. Oxford, Clarendon Press.

\section{Magnusson, Sven-Erik}

2004 "The changing perception of the wetlands in and around Kristianstad, Sweden: From waterlogged areas toward a future water kingdom, Kristianstads Vattenrike Biosphere Reserve ", Annals of the New York Academy of Sciences 1023: 323-327.

\section{Mairesse, François}

2012 Le Musée hybride. Paris, La Documentation française.

\section{Martin, Jean-Hubert, Guillaume, Jean et Didier, Frédéric}

2000 Le Château d'Oiron et son cabinet de curiosités. Paris, MONUM-Centre des monuments nationaux-Éditions du patrimoine.

\section{Martin, Jean-Hubert}

2013 "Théâtre du monde: le musée des charmes versus le musée docile", in Collectif, Théâtre du monde. Lyon et Paris, Fage Éditions-La Maison rouge: 13-26.

\section{Meijers, Debora}

2001 «La classification comme principe: la transformation de la Galerie impériale de Vienne en "histoire visible de l'art" ", in Édouard Pommier (dir.), Les Musées en Europe à la veille de l'ouverture du Louvre. Paris, Klincksieck: 591-605.

\section{Meyer, Morgan}

2009 «Objet-frontière ou projetfrontière? Construction, (non-) utilisation et politique d'une banque de données ", Revue d'anthropologie des connaissances 3(1): 127-148.

\section{Moreno, Francisco P.}

1890-1891 «El museo de La Plata. Rápida ojeada sobre su fundación y desarrollo ", Revista del Museo de La Plata 1 : 28-70.

\section{Nicklin, Keith}

2001 «Quex for adventure: The Powell-Cotton family enterprise from field to showcase", in Anthony Shelton (éd.), Collectors. Expressions of Self and Others. Londres, Horniman Museum : 147-156.

\section{Olalquiaga, Céleste}

2002 Royaume de l'artifice. L'émergence du kitsch au XIx siècle. Lyon, Fage Éditions.

\section{Panese, Francesco}

2003 "Les régimes muséologiques dans le domaine des sciences", in Béatrice Pellegrini (dir.), Sciences au musée, sciences nomades. Genève et Paris, Georg Éditeur : 7-28.

\section{Pearce, Susan}

1992 Museums, Objects and Collections: A Cultural Study. Leicester, Leicester University Press.

\section{Pomian, Krzysztof}

1988 "Musée archéologique: art, nature, histoire ", Le Débat 49: 57-68.

1993 Collectionneurs, amateurs et curieux. Paris, Venise: $X V I^{e}-X V I I^{e}$ siècles. Paris, Gallimard.

2001 "Collection: une typologie historique ", Romantisme 112: 9-22.

2004 «Histoire naturelle: de la curiosité à la discipline ", in Pierre Martin et Dominique Moncond'huy (dir.), Curiosité et cabinets de curiosités. Neuilly, Atlante: 15-40.

\section{Poulot, Dominique}

2011 "L'histoire des collections, une histoire anecdotique? ", in Odile Vincent (dir.), Collectionner? Territoires, objets, destins. Paris, Créaphis Éditions : 7-17.

\section{Rivière, Georges Henri}

1971 "Le musée de plein air des Landes de Gascogne ", Ethnologie française n.s.1(1) : 87-95.

\section{Schnapper, Antoine}

1994 Curieux du Grand Siècle. Collections et collectionneurs dans la France du xvil siècle, vol. II: CEuvres d'art. Paris, Flammarion.

\section{Seipel, Wilfried (éd.)}

2000 Exotica. Portugals Entdeckungen im Spiegel fürstlicher Kunst- und Wunderkammern der Renaissance. Vienne, Kunsthistorisches Museum-Skira Verlag.
Star, Susan Leigh et Griesemer, James R.

1989 «Institutional ecology, "translations", and boundary objects: Amateurs and professionals in Berkeley's Museum of Vertebrate Zoology ", Social Studies of Science 19(3) : 387-420.

\section{Stocking, George W. Jr}

1974 The Shaping of American Anthropology, 1883-1911. A Franz Boas Reader. New York, Basic Books.

\section{Strasser, Bruno}

2012 "Collecting nature: Practices, styles, and narratives ", Osiris 27(1): 303-340.

\section{Trachsel, Albert}

1898 Réflexions sur l'enseignement. Paris, Librairie de la France scolaire.

\section{Trompette, Pascale et Vinck, Dominique}

2009 «Retour sur la notion d'objetfrontière ", Revue d'anthropologie des connaissances (3)1: 5-27.

\section{Vergnioux Alain}

2010 "Conférence de M. Bagnaux sur les musées scolaires à l'Exposition universelle de 1878 ", Les Sciences de l'éducation. Pour l'Ére nouvelle 43(4): 123-129.

page 28 et ci-contre

Installation de l'artiste Rosamond Purcell, 2013, reprenan le cabinet de curiosité d'Ole Worm au Museum Wormianum en 1655 Photo Jens Astrup, Natural History Museum of Denmark. 